\title{
THE EFFECTS OF AGING AND DEGENERATIVE VASCULAR DISEASE ON THE MEASUREMENT OF ARTERIAL RIGIDITY IN MAN*
}

\author{
By FRANCOIS M. ABBOLD $\dagger$ AND JOHN H. HUSTON \\ (From the Cardiovascular Section, Department of Medicine, Marquette University School of \\ Medicine and the Milz'aukee County Hospital, Milz'auke, Wis.)
}

(Submitted for publication December 6, 1960; accepted February 9, 1961)

In 1937, Hallock and Benson demonstrated a progressive loss of aortic distensibility with aging (1). Their observations on the pressure-volume relationship in segments of human aortas from five age groups are shown in Figure 1 . The curves which represent the less distensible older aortas have a greater slope $(\mathrm{d} P / \mathrm{d} \mathrm{V})$. The slope of each curve is not fixed, however, and it changes by bending toward the pressure axis as pressure increases. The rate of this change in slope (or the curvature) also is greater in the curves of older aortas.

The present report deals with an indirect clinical index of the curvature of arterial pressurevolume curves, using the ratio of the change in pulse pressure to the change in diastolic pressure following the inhalation of amyl nitrite. This index is referred to, arbitrarily, as one of "arterial rigidity." A study of Hallock and Benson's curves made it apparent to us that the change in the slope of each curve is associated with a corresponding change in pulse pressure at different diastolic pressures. If the pressure-volume curve is relatively straight, as it is in the young, the curvature and the index will be near zero (Figure 2). If the line curves greatly toward the pressure axis, the index will be positive and large (Figure 3 ). If it curves the other way, the index will be negative. In vitro indices were derived from each of the five aortic distensibility curves and were found to correlate with age (Figure 4). This observation suggested that the values of this index in "normal" subjects also should increase with chronological age. The present investigation was undertaken to test this hypothesis.

* Supported by a research grant from the American Heart Association.

† Work done during tenure of an American Heart Association Research Fellowship. Present address : Cardiovascular Research Laboratories, College of Medicine, State University of Iowa, Iowa City, Iowa.

\section{MATERIALS AND METHODS}

Selection of subjects. The subjects for this investigation were patients from the wards and members of the professional staff of Milwaukee County Hospital. One hundred fifteen subjects varying from 8 to 95 years of age were studied. They were separated into nine age groups. Seven subjects, aged 8 to 19 years, comprised the first group; 4 subjects were 20 to 29 years of age; 18 were 30 to 39 years; 24 were 40 to 49 years; 21 were 50 to 59 years; 13 were 60 to 69 years; 16 were 70 to 79 years ; 9 were 80 to 89 years; and 3 were 90 to 95 years. A careful clinical examination and review of the hospital records disclosed normal retinal vessels, ${ }^{1}$ "normal" blood pressures ${ }^{2}$ and freedom from heart disease, overt periph-

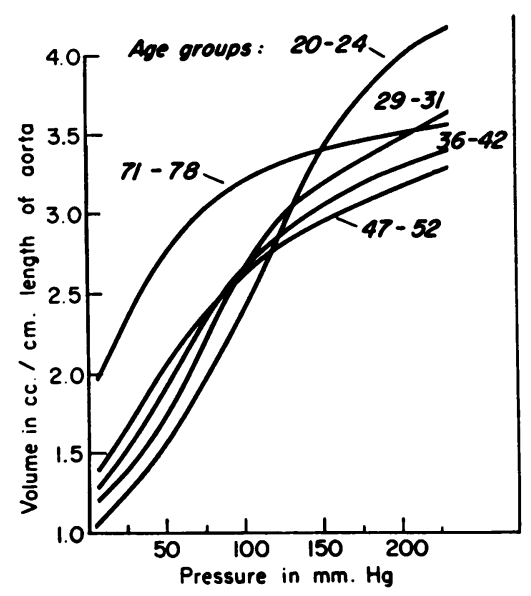

Fig. 1. Pressure-volume curves of segments of HUMAN AORTAS FROM FIVE AGE GROUPS (FROM THE EXPERIMENTAL data of Hallock and Benson) (1). The curve which represents the older aorta has a greater slope $(\mathrm{d} \mathrm{P} / \mathrm{d} \mathrm{V})$. It also bends toward the pressure axis with a greater curvature (in the operating pressure range) than do the curves of younger aortas. (Permission to reprint this figure from the J. clin. Invest. 1937, 16, 599 was granted by the editor.)

${ }^{1}$ Silver wiring and tortuosity were accepted as normal retinal changes in subjects over 60 years of age.

2 All the subjects under 60 years of age had blood pressures below 140/80 and all those over 60 years had blood pressures below $170 / 90$. 


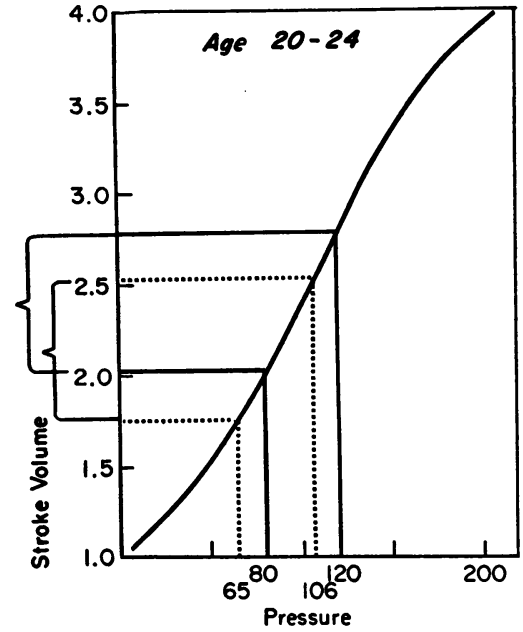

Fig. 2. Pressure-volume curve of human aorta (AGE 20 To 24 yeARS) FROM Figure 1. Two equivalent stroke outputs were plotted on the ordinate so that their corresponding pulse pressures on the abscissa were within the normal range for the age group and the difference between their diastolic pressures was approximately $15 \mathrm{~mm}$ $\mathrm{Hg}$. Note that because the pressure-volume curve is relatively straight there is little change in pulse pressure for the same stroke volume at various pressure levels. The calculation of the rigidity index (fall in pulse pressure $\times 100 /$ fall in diastolic pressure) reveals a slight increase in pulse pressure at a lower diastolic level resulting in a small negative value of -7 .

eral vascular disease and diabetes. None had a family history of diabetes. Roentgenographic examinations of their chests were normal except for calcification or tortuosity of the aorta in 6 of 28 persons over 70 years of age. Electrocardiograms revealed no significant abnormalities. This group constituted the "normal" subjects used to determine the effect of aging on arterial rigidity.

Twenty-five other patients were selected because of clinical evidence of arteriosclerotic vascular disease. An attempt was made to correlate their arterial rigidity indices with various overt manifestations of arteriosclerosis. Eight of them had intermittent claudication (arteriosclerosis obliterans); 5 had had cerebrovascular accidents; 5 had had myocardial infarctions; 3 appeared prematurely senile and had angina pectoris; 2 were myxedematous; 1 had an aortic arch syndrome with a hard nodular carotid artery; and 1 had a calcified aortic stenosis.

Method. The arterial rigidity index was determined in all subjects. The method was originally described by Conway and Smith (2) and its theoretical background has been discussed. It requires accurate measurement of the blood pressure changes immediately following amyl nitrite inhalation. To be valid it requires a nearly constant left ventricular stroke output during the blood pressure measurement. There is evidence that only an insignificant change in stroke output occurs during the brief period of hypotension which follows the inhalation of amyl nitrite and precedes the onset of reflex tachycardia (2-4). The ratio of the change in pulse pressure times 100 to the related decrease in diastolic pressure during this period gives the index of arterial rigidity. In older subjects with more rigid arteries there was a greater fall in pulse pressure and consequently a higher index.

The nature and purpose of the procedure were discussed with the subjects. They received no premedication and were allowed to rest comfortably on their backs for 10 minutes before the test. Under local infiltration anesthesia, a Cournand needle was introduced into the brachial artery and connected with a short piece of polyethylene tubing (internal diameter, $1 \mathrm{~mm}$ ) to a strain gage pressure transducer. The resulting signal was amplified and recorded ${ }^{3}$ at a paper speed of $25 \mathrm{~mm}$ per second. The transducer-recorder system was calibrated against known static pressures and adjusted to give maximal deflections for each blood pressure recording. The systemic arterial pressure, electrocardiogram, and respiratory movements were recorded simultaneously. Respiratory movements were recorded by fitting a hollow rubber tube around the chest and attaching it to a pressure transducer. After establishing control values, a crushed vial of amyl nitrite was held just under the subject's nose during 1 or 2 normal inspirations and he or she was instructed to maintain a constant respiratory pattern without talking, coughing, or sniffing the vapor. Analysis of the pressure tracings involved selec-

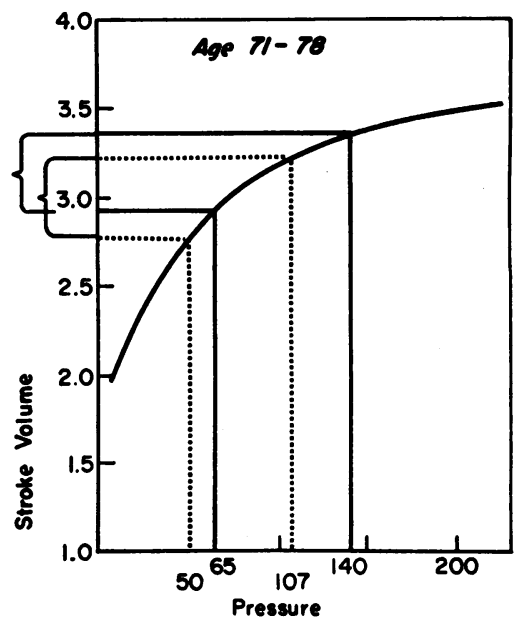

Fig. 3. Pressure-volume curve of human aorta (AgE 71 to 78 years) From Figure 1 . Because the slope bends greatly toward the pressure axis as the pressure is increased, there is a marked drop in pulse pressure for the same stroke volume at a lower diastolic pressure. The rigidity index, calculated as described in Figure 2, was +120 .

\footnotetext{
${ }^{3}$ Sanborn multiple-channel recorder.
} 


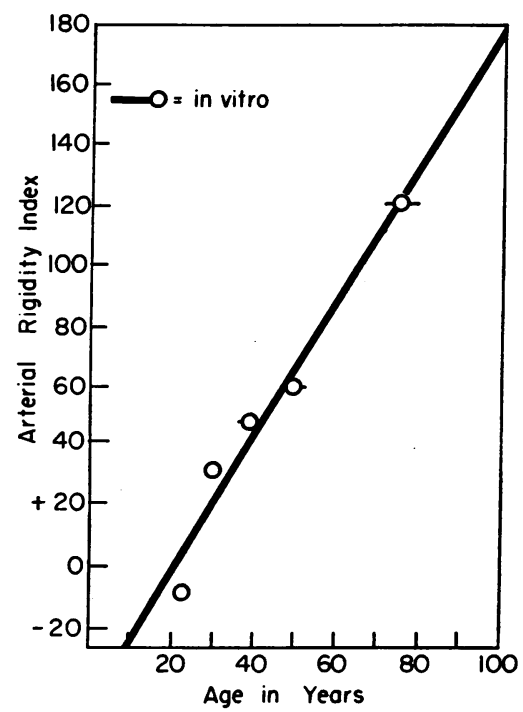

Fig. 4. IN VITRO ARTERIAL RIGIDITY INDICES CALCULATED FROM EACH OF THE FIVE PRESSURE VOLUME CURVES of Hallock and Benson USING the METHOD DESCRIBED IN FIGURE 2. Note the progressive increase of these indices with age $(\mathrm{R}=0.977, \mathrm{p}=<0.005)$.

tion of equivalent beats in respect to heart rate and phase of respiration before the inhalation of amyl nitrite and immediately after its hypotensive effect. Seven to 10 beats usually were available for study before reflex tachycardia occurred (Figure 5). A decrease in diastolic pressure greater than $7 \mathrm{~mm} \mathrm{Hg}$ was judged arbitrarily to be necessary for a sufficiently accurate calculation. Consistent results were obtained in each of three subjects in whom the test was repeated during a second session. The regression and correlation of the indices with aging were determined (6). An equation for the upper limit of normal was calculated at +2 sample standard deviations from the regression.

Rigidity indices also were calculated from five in vitro aortic pressure-volume curves of Hallock and Benson (Figure 1). These curves were derived from experimental observations made on isolated segments of thoracic aortas from persons in the age groups 20 to 24,29 to 31 , 36 to 42,47 to 52 and 71 to 78 years. As far as could be determined, all individuals but those of the last group were normal. Those beyond 70 years showed a minimal degree of coronary disease. Figures 2 and 3 illustrate the method used to calculate the indices from the curves. Two equivalent stroke outputs were plotted on the ordinate so that their corresponding pulse pressures were within the normal range for the age group and the difference between their diastolic pressures was approximately $15 \mathrm{~mm} \mathrm{Hg}$. The indices were calculated as they were in vivo. The regression and correlation of the in vitro indices with age were calculated and compared with the in vivo data.
RESULTS

Effect of chronological aging on the arterial rigidity index. The mean arterial rigidity indices for the "normal" groups according to increasing age were: $-10.7,+1.5,+17.5,+42.5,+53.5$, $+99.6,+112.6,+141.6$, and +121.0 (Table I). Six subjects in the first group ( 8 to 19 years) had an increase in the pulse pressure as the diastolic pressure fell and the mean arterial rigidity was thus expressed with a negative sign. A significant positive correlation $(R=0.937, p=<0.001)$ between the indices and aging was obtained. Fig-
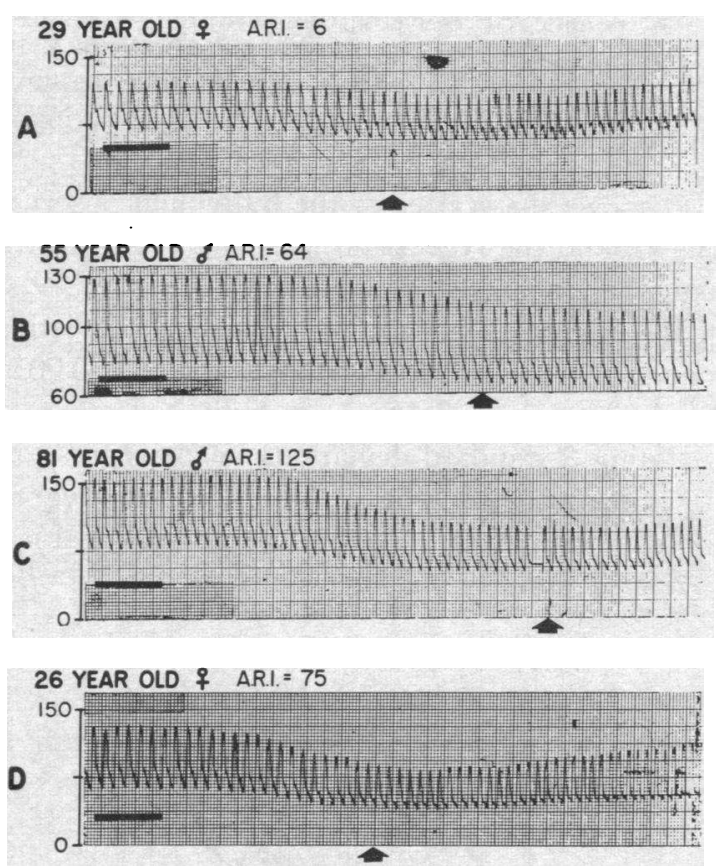

Fig. 5. Brachial ARTERIal PRESSURES RECORDED BEFORE, DURING AND AFTER AMYL NITRITE INHALATION IN FOUR SUBJECTS. The time of inhalation of amyl nitrite is indicated by the horizontal lines below the tracings. The vertical arrows indicate the onset of reflex tachycardia. A brief period of hypotension precedes the onset of reflex tachycardia. Pressures from this period were used to calculate the arterial rigidity index, A.R.I. Note that the fall in pulse pressure which is insignificant in the young individual (A), progresses with age resulting in higher indices in (B) and (C). A young patient (D) with premature arteriosclerosis had a pressure response comparable to that of older subjects. Note the disappearance of the dicrotic notch in the older subject (C) and in the prematurely arteriosclerotic subject (D). This coincides with the abnormality in dicrotic contours observed by Lax, Feinberg and Cohen (5). 
TABLE I

Effect of amyl nitrite inhalation on the blood pressure of 115 "normal" subjects and the calculated mean arterial rigidity index in each age group *

\begin{tabular}{|c|c|c|c|c|c|c|c|}
\hline \multirow{3}{*}{$\begin{array}{c}\text { Age } \\
\text { groups }\end{array}$} & \multirow{3}{*}{$\begin{array}{l}\text { No. of } \\
\text { subjects } \\
\text { and Sex }\end{array}$} & \multicolumn{4}{|c|}{ Average brachial artery pressures } & \multirow{3}{*}{$\begin{array}{l}\text { Average } \\
\text { change } \\
\text { in PP } \\
\text { after } \\
\text { nitrite }\end{array}$} & \multirow{3}{*}{$\begin{array}{c}\text { Average } \\
\text { rigidity } \\
\text { indext }\end{array}$} \\
\hline & & \multicolumn{2}{|c|}{ Before nitrite } & \multicolumn{2}{|c|}{ After nitrite } & & \\
\hline & & SP & $\overline{\mathrm{DP}}$ & SP & $\mathrm{DP}$ & & \\
\hline yrs & & \multicolumn{2}{|c|}{$m m ~ H g$} & \multicolumn{2}{|c|}{$m m ~ H g$} & $m m \mathrm{Hg}$ & \\
\hline $8-19$ & $7(5 \mathrm{M})$ & 111.8 & 65.8 & 98.5 & 50.9 & $+1.6 t$ & $-10.7 t$ \\
\hline $20-29$ & $4(2 \mathrm{M})$ & 127.2 & 77.0 & 113.3 & 63.3 & $-0.2^{+}$ & $\begin{array}{l}1.5^{+} \\
+\quad 1 .{ }^{\circ}\end{array}$ \\
\hline $30-39$ & $18(9 \mathrm{M})$ & 124.5 & 70.6 & 108.9 & 57.3 & $\begin{array}{l}0.2 \\
-\quad 2.3\end{array}$ & $\begin{array}{l}17.5 \\
+\quad 17.5\end{array}$ \\
\hline $40-49$ & $24(15 \mathrm{M})$ & 130.6 & 70.0 & 111.5 & 56.6 & $\begin{array}{r}2.0 \\
-5.7\end{array}$ & $\begin{array}{r}1.5 \\
+\quad 42.5\end{array}$ \\
\hline $50-59$ & $21(15 \mathrm{M})$ & 131.1 & 70.8 & 111.0 & 57.7 & $\begin{array}{r}-7.0 \\
-7.0\end{array}$ & $\begin{array}{r}+5 . .5 \\
+\quad 53.5\end{array}$ \\
\hline $60-69$ & $13(9 \mathrm{M})$ & 142.3 & 72.0 & 120.1 & 60.9 & -11.1 & $\begin{array}{r}09.6 \\
+\quad 99.6\end{array}$ \\
\hline $70-79$ & $16(11 \mathrm{M})$ & 143.0 & 66.7 & 114.3 & 53.2 & -15.2 & $\begin{array}{r}+112.6 \\
+112.0\end{array}$ \\
\hline $80-89$ & $9(5 \mathrm{M})$ & 146.8 & 61.5 & 115.4 & 48.5 & -18.4 & +141.6 \\
\hline $90-95$ & $3(2 \mathrm{M})$ & 167.1 & 75.0 & 128.4 & 57.5 & -21.2 & +121.0 \\
\hline
\end{tabular}

* $\mathrm{M}=$ male $; \mathrm{SP}=$ systolic pressure $; \mathrm{DP}=$ diastolic pressure $; \mathrm{PP}=$ pulse pressure.

$\dagger$ Arterial rigidity index $=\frac{\text { fall in pulse pressure } \times 100}{\text { fall in diastolic pressure }}$.

$\ddagger$ There was an increase in pulse pressure as the diastolic pressure fell in this group only and the index was expressed with a negative sign.

ure 6 shows the scatter of the individual observations around the regression line which was drawn from the calculated equation: index $=(2.19 \times$ age) -53.84 . The sample standard deviation from this regression was $\pm 16.16(\mathrm{p}=<0.001)$ and the equation of the upper limit was obtained by adding 2 standard deviations to the regression, i.e., index $=(2.19 \times$ age $)-21.52$. The regression coefficient was $2.19 \pm 0.15(\mathrm{p}=<0.001)$.

Arterial rigidity indices calculated from in vitro

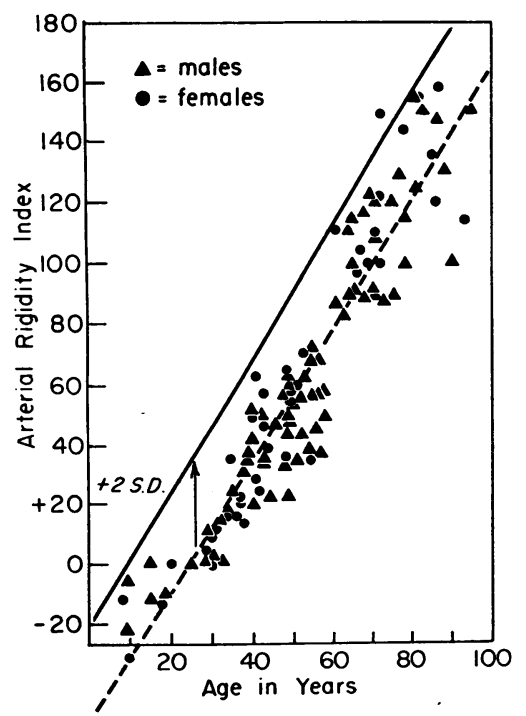

Fig. 6. Arterial Rigidity indices (ordinate) of 115 "NORMAL" SUBJECTS RANGING FROM 8 TO 95 YEARS OF AGE. The upper limit of normal (solid line) is drawn at $+2 \mathrm{SD}$ from the regression line (broken line). pressure-volume curves of normal human aortas. The indices, calculated from the five curves in Figure 1, according to increasing age were -10 , $+33,+49,+60$ and +120 , respectively. Figure 4 shows the strong correlation $(\mathrm{R}=0.977$, $\mathrm{p}=<0.005)$ between the indices and age with the regression line drawn according to the calculated equation: index $=(2.26 \times$ age $)-46.78 \pm$ $11.51(\mathrm{p}=<0.005)$. The regression coefficient was $2.26 \pm 0.28(\mathrm{p}=<0.005)$.

Comparison between in vivo and in vitro data. Figure 7 shows the close agreement between the mean indices determined clinically and those derived in vitro. The regressions of the two groups of data with age were very similar as were their regression coefficients.

Arterial rigidity indices in patients with clinical evidence of arteriosclerosis. The 25 patients in this group had signs and symptoms of arteriosclerotic vascular disease involving various portions of their arterial system. Twenty-three of them had indices that were greater than the upper limits of normal for their age (Figure 8). The usual clinical manifestations of arteriosclerotic vascular disease (advanced arteriosclerotic retinal changes, calcifications or tortuosity of the aorta, and systolic hypertension ${ }^{4}$ ) were found in only 17 of these 23 patients.

${ }^{4}$ A systolic hypertension was considered present when the diastolic pressure was normal and the pulse pressure was $100 \mathrm{~mm} \mathrm{Hg}$ or more. 


\section{DISCUSSION}

Conway and Smith described the ratio of the fall in pulse pressure to the fall in diastolic pressure following the inhalation of amyl nitrite as an index of "arterial elasticity" (2). In a study of aging of the arteries in relation to hypertension (7), they found that the lack of "arterial elasticity" in a group of older hypertensive subjects significantly exceeded that in 16 young "normal" medical students and concluded that hardening of the arteries may be one cause for diastolic hypertension. Because of the well known correlation of arteriosclerosis with chronological aging, we suspected that a number of the hypertensives in their studies might be considered to have "normal elasticity" when compared with "normal" subjects of their same age. A clear understanding of what this index represents and a knowledge of the effect of aging on its values are needed.

A close study of Hallock and Benson's pressurevolume curves indicated that the index is not measuring the slope of these curves but the rate of change of the slope (or the curvature) with pressure. Thus, it is not directly related to the distensibility of the large arteries but it represents

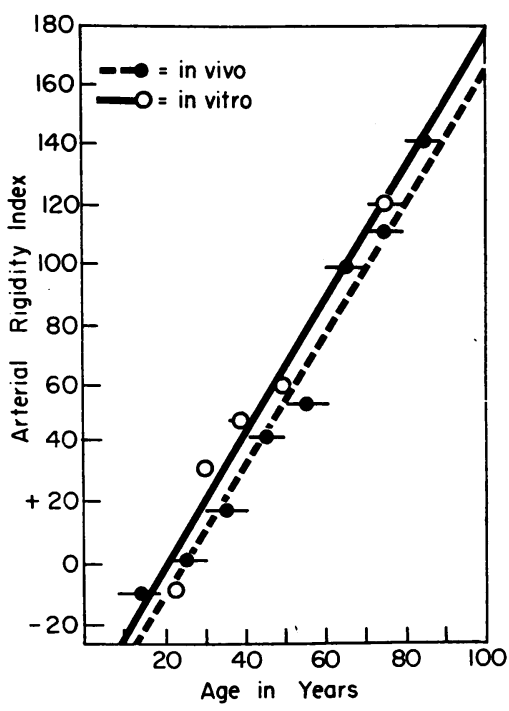

Fig. 7. Note the Close AgReEMent Between the IN VITRO INDICES (OPEN CIRCLES) AND THE MEAN VALUES OBTAINED WITH THE AMYL NITRITE TEST IN 115 "NORMAL" SUBJECTS (DOTS). The regression equations and regression coefficients of the 2 groups of data (in vitro and in vivo) were very similar.

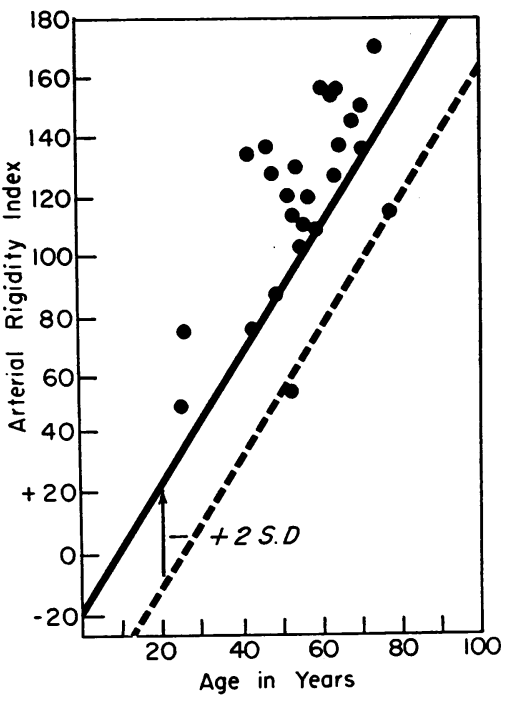

Fig. 8. Arterial Rigidity indices (ORdinate) of 25 PATIENTS WITH CLINICAL EVIDENCE OF ARTERIOSCLEROSIS INVOLVING VARIOUS PORTIONS OF THE ARTERIAL SYSTEM. The solid line represents the upper limit of normal as in Figure 6. Note that 23 arteriosclerotic individuals had arterial rigidity indices which exceeded the upper limit of normal for their age group.

the differential of their stiffness ${ }^{5}$ with respect to pressure. Its usefulness, in spite of its indirectness, results entirely from the empiric fact that the classical curves of Hallock and Benson show a progressive increase in their curvature with age. This relationship between the index and the curvature brings up three important possibilities. The first is that the index should increase with age since the "curvature" increases with age. This is demonstrated both in vivo and in vitro in this report. The second possibility is that the index provides an indirect estimate of the number and state of collagenous and elastin fibers in the large arteries. Roach and Burton showed that the shape of arterial pressure-volume curves results from the combined effect of the elastin and collagenous fibers in the vessel wall (9). They proposed that the increased curvature seen in the distensibility curves of old arteries represents an increase in collagenous material and a decrease in

${ }^{5}$ Stiffness (sometimes defined as a secant modulus) has been used to describe behavior of biological materials without reference to cross sectional area and initial length measurements. In this sense stiffness is defined as the ratio of increment in tension to increment in length (8). 
the unstretched length of the collagenous fibers as the "slack" in them is taken up, possibly by cross linkages and adhesions (10). The third possibility is that the index may be invalid at pressure levels outside the "normal" range (170 to $55 \mathrm{~mm} \mathrm{Hg}$ ), since the progressive increase in curvature with age occurs only within this pressure range (Figure 1). At pressures higher than $200 \mathrm{~mm} \mathrm{Hg}$ the curves of the older aortas are straight while those of the younger vessels are still bending toward the pressure axis.

The results obtained in 115 "normal" subjects provide the needed information for a more correct interpretation of the results of the test in patients of all age groups. In order to check the quantitative validity of the in vivo "normal" values we compared them with the in vitro indices derived from the aortic pressure-volume curves of Hallock and Benson in various age groups. The agreement between the two groups of data was very close (Figure 7). It is apparent from these observations that the method provides a quantitative measurement of the "rigidity" of large arteries.

Our results in 23 of the 25 patients with overt premature arteriosclerosis demonstrate the effect of superimposed degenerative arterial disease on the "rigidity" of aging. The upper limit of normal for this test separates these patients from "normal" individuals of the same age (Figure 8). Only two patients presumed to have arteriosclerosis because of a previous history of myocardial infarction were found to have normal rigidity indices. Such a finding is not surprising, since coronary artery occlusions not uncommonly occur in subjects whose larger arteries do not show significant arteriosclerosis. The older of the two patients was 77 years old and had evidence of aortic tortuosity on the roentgenogram.

Various important applications of this test might be considered. The first is a clinical estimation of the severity of arterial rigidity. Previously, attempts to quantitate this vascular change had been made by measurements of arterial pulse wave velocity $(1,11)$. This method proved to be of limited clinical value because of the difficulty in measuring the distance over which the pulse wave traveled, especially when arteries were elongated and tortuous. The amyl nitrite test obviates this difficulty. It also may be used to confirm a clini- cal suspicion of premature arteriosclerosis, particularly in patients who do not yet manifest the usual signs.

As an investigative tool this test might clarify the relationship between arteriosclerosis and the different types of hypertension or the various aspects of the diabetic state. Further, long-term studies of the effect of environmental, dietary and hormonal factors on the "normal" regression of arterial rigidity with age might yield some very useful information.

\section{SUM MARY}

A clinical index of arterial rigidity was investgated. In previous studies the upper limit of normal for this index was obtained from the data on young medical students. Because arteriosclerosis progresses with age, the normal values for this index also should increase with age if it reflects arterial rigidity. Our data on 115 "normal" subjects demonstrated a strong positive correlation of this index with age and were in agreement with in vitro indices calculated from pressure-volume curves of normal human aortas in similar age groups. An equation representing the upper limit of normal separated the arterial rigidity of aging from that of premature arteriosclerosis. The results suggest that this test offers more than just a qualitative estimate of arteriosclerosis.

With a knowledge of the variation of normal values with age, the applicability of this test can be extended to all age groups and a more correct interpretation of previous studies utilizing this index can be made.

\section{ACKNOWLEDGMENT}

The authors wish to thank Dr. John W. Eckstein for his advice and suggestions in the preparation of this manuscript.

\section{REFERENCES}

1. Hallock, P., and Benson, I. C. Studies on the elastic properties of human isolated aorta. J. clin. Invest. 1937, 16, 595.

2. Conway, J., and Smith, K. S. A clinical method of studying the elasticity of large arteries. Brit. Heart J. 1956, 18, 467.

3. Conway, J. Direct and reflex effects of injections of nitroglycerine and L-noradrenaline in normal and hypertensive rabbits. Clin. Sci. 1955, 14, 625. 
4. Abboud, F., and Huston, J. Unpublished observations.

5. Lax, H., Feinberg, A. W., and Cohen, B. M. Studies of the arterial pulse wave. I. The normal pulse wave and its modification in the presence of human arteriosclerosis. J. chron. Dis. 1956, 3, 618.

6. Snedecor, G. W. Statistical Methods Applied to Experiments in Agriculture and Biology, 5th ed. Ames, Iowa, State College Press, 1956, pp. 122-193.

7. Conway, J., and Smith, K. S. Aging of arteries in relation to hypertension. Circulation 1957, 15, 827.
8. Remington, J. W., Ed. Tissue Elasticity. Washington, American Physiological Society, 1957, p. 197.

9. Roach, M. R., and Burton, A. C. The reason for the shape of the distensibility curves of arteries. Canad. J. Biochem. 1957, 35, 681.

10. Roach, M. R., and Burton, A. C. The effect of age on the elasticity of human iliac arteries. Canad. J. Biochem. 1959, 37, 557.

11. Hallock, P. Arterial elasticity in man in relation to age as evaluated by the pulse wave velocity method. Arch. intern. Med. 1934, 54, 770.

\section{CORRECTION}

On page 868 of the article entitled "Sodium Depletion and Renal Conservation of Water" by Geoge P. Baker, Howard Levitin and Franklin H. Epstein (J. clin. Invest. 1961, 40, 867), the italicized subheading under "Bone $\mathrm{Na}$ " in Table II should read " $m E q / g$ dry solids." This had been incorrectly printed as " $m E q / 100$ g dry solids." 